\title{
ON A NEW TYPE OF $\ell$-ADIC REGULATOR FOR ALGEBRAIC NUMBER FIELDS. II
}

\author{
L. V. KUZ'MIN \\ Dedicated to S. V. Vostokov \\ on the occasion of his 70 th anniversary.
}

\begin{abstract}
In a preceding paper of the author, a new type of an $\ell$-adic regulator $\mathfrak{R}_{\ell}(K)$ was introduced for an algebraic number field $K$ such that the prime $\ell$ splits completely in $K$. Nevertheless, the element $\mathfrak{R}_{\ell}(K) \in \mathbb{Z}_{\ell}$ is defined only up to an arbitrary factor in $\left(\mathbb{Z}_{\ell}^{\times}\right)^{2}$. In the present paper, under the assumption of the validity of the Shanuel conjecture (both Archimedean and $\ell$-adic), the definition of $\mathfrak{R}_{\ell}(K)$ as a certain number in $\mathbb{Z}_{\ell}$ is given. For a real quadratic field $K$, such a defition can be obtained without using any additional conjectures.
\end{abstract}

\section{$\S 1$. INTRODUCTION}

Let $K$ be an algebraic number field and $\ell$ a fixed prime number. Let $U(K)$ be the group of units of $K$. For the field $K$, there is a classical (Archimedean) regulator, which will be denoted by $R_{\infty}(K)$ in this paper (see [1, Chapter 2]). This regulator is defined in terms of embedding of the group $U(K)$ into the logarithmic space. Similarly, one can define the Leopoldt $\ell$-adic regulator $\mathbf{R}_{\ell}(K)$ (see [2]). Unfortunately, this regulator is well defined only for totally real $K$.

As is well known, $R_{\infty}(K) \neq 0$ for any algebraic number field $K$. It is conjectured that $\mathbf{R}_{\ell}(K)$ is also nonzero for any prime $\ell$ and any totally real $K$. Herein, the relation $\mathbf{R}_{\ell}(K) \neq 0$ is equivalent to the validity of the Leopoldt conjecture.

In [3], the author proposed a new definition of an $\ell$-adic regulator, which makes sense for any algebraic number field. (We shall denote this regulator by $R_{\ell}(K)$.) This new regulator turned out to be useful and yielded some interesting consequences even in the case of totally real fields [5].

Note that $R_{\infty}(K)$ is a uniquely defined positive real number. The Leopoldt regulator $\mathbf{R}_{\ell}(K)$ is an element of the algebraic closure $\overline{\mathbb{Q}}_{\ell}$ of the rational $\ell$-adic number field $\mathbb{Q}_{\ell}$ and is defined uniquely up to a sign. The regulator $R_{\ell}(K)$ is a uniquely defined element of the field $\mathbb{Q}_{\ell}$. (This fact combined with [5, Proposition 2.1] shows that $\mathbf{R}_{\ell}(K)$ belongs to some quadratic extension of $\mathbb{Q}_{\ell}$ depending on $K$.) Note that the regulators $R_{\infty}(K), \mathbf{R}_{\ell}(K)$, and $R_{\ell}(K)$ are defined in terms of some fundamental system of units $\varepsilon_{1}, \ldots, \varepsilon_{r}$ of $K$, where $r$ is the rank of $U(K)$. If $\varepsilon_{1}, \ldots, \varepsilon_{r}$ and $\varepsilon_{1}^{\prime}, \ldots, \varepsilon_{r}^{\prime}$ are two such systems linked by some transition matrix $C \in \mathrm{GL}(r, \mathbb{Z})$, then $\operatorname{det} C= \pm 1$, and the regulators $R_{\infty}(K)$ defined by these two systems differ by multiplication by $|\operatorname{det} C|=1$, the regulators $\mathbf{R}_{\ell}(K)$ differ by multiplication by $\operatorname{det}(C)= \pm 1$, and, finally, the regulators $R_{\ell}(K)$ differ by multiplication by $\operatorname{det}(C)^{2}=1$.

2010 Mathematics Subject Classification. Primary 11R04.

Key words and phrases. $\ell$-adic regulator, $S$-units, global universal norms, Shanuel conjecture, Iwasawa theory.

The author was supported by RFBR (grant no. 14-01-00393). 
In [4], the author introduced a new $\ell$-adic regulator $\mathfrak{R}_{\ell}(K)$, which is suitable for applications related to the Iwasawa theory. Let $K_{\infty} / K$ be the cyclotomic $\mathbb{Z}_{\ell^{-}}$extension of $K, K_{\infty}=\cup_{n} K_{n}$, where $\left[K_{n}: K\right]=\ell^{n}$, and $U_{S}\left(K_{n}\right)[\ell]$ the pro- $\ell$-completion of the group $U_{S}\left(K_{n}\right)$ of $S$-units of $K_{n}$, where $S$ is the set of all places over $\ell$ in the field under consideration. Let $U_{S, 1}(K)$ be the subgroup of global universal norms in $U_{S}(K)[\ell] / \mu_{\ell}(K)$, where $\mu_{\ell}(K)$ is the group of all roots of unity of $\ell$-power degree in $K$, that is,

$$
U_{S, 1}(K)=\bigcap_{n=1}^{\infty}\left(N_{K_{n} / K}\left(U_{S}\left(K_{n}\right)[\ell] / \mu_{\ell}\left(K_{n}\right)\right)\right) .
$$

The regulator $\mathfrak{R}_{\ell}(K)$, which will be defined in more detail in the next section, characterizes the $\mathbb{Z}_{\ell}$-module $U_{S, 1}(K)$ and is defined in terms of a $\mathbb{Z}_{\ell}$-basis of that module. Thus, the element $\mathfrak{R}_{\ell}(K) \in \mathbb{Z}_{\ell}$ is defined up to multiplication by some element $c^{2}$, where $c \in \mathbb{Z}_{\ell}^{\times}$. The following question 1 arises: Can the definition of this regulator be refined so as to obtain $\mathfrak{R}_{\ell}(K)$ as a uniquely defined number, as in the case of the regulator $R_{\ell}(K)$ ?

Our goal here is to answer this question in the positive under the assumption that the Shanuel conjecture holds true (both $\ell$-adic and Archimedean; see Conjectures 1 and 2 in $\S 2)$. First, we assume $K$ to be normal over $\mathbb{Q}$. In the definition of $\mathfrak{R}_{\ell}(K)$ in 4 ] we used an element $\alpha \in K^{\times}$such that $(\alpha)=\mathfrak{l}_{1}^{\mathbf{h}}$, where $\mathfrak{l}_{1}$ is a fixed prime divisor of $\ell$ in $K$ and $\mathbf{h}$ is the order of $\mathfrak{l}_{1}$ in the class group. Such an element is defined up to multiplication by an arbitrary unit of $U(K)$. So, the first problem we meet is the choice of a canonical representative $\widetilde{\alpha}$ in the set $\alpha U(K)$. We prove (see Theorem 4 ) that this can be done, assuming that the Arcihmedean Shanuel conjecture is valid.

In $\S 3$, we define a new group $U_{S, 3}^{0}(K)$ intimately related to $U_{S, 1}(K)$ and, using the element $\widetilde{\alpha}$ and some fundamental system of units in $K$, construct a "good" basis in $U_{S, 3}^{0}(K)$. Then we prove that one can pass from one "good" basis to another via a transition matrix $C \in \mathrm{GL}(r, \mathbb{Z})$. Hence, any "good" basis determines the same regulator $\mathfrak{R}_{\ell, 3}^{0}(K)$ of the group $U_{S, 3}^{0}(K)$. Then it is easy to pass to the group $U_{S, 1}(K)$ and its regulator $\mathfrak{R}_{\ell}(K)$. The precise definitions are given in (9) and (10). All these definitions can easily be carried over to the case of a nonnormal extension of $\mathbb{Q}$ (see (12) and (13)).

For a real quadratic $K$ we prove (see Theorem 6 ) that the existence of the element $\widetilde{\alpha}$ can be established without using any additional conjectures.

\section{§2. Preliminary Results}

As in 4], we assume that the following particular case of the $\ell$-adic Shanuel conjecture holds true.

Conjecture 1. Suppose that the numbers $x_{1}, \ldots, x_{n} \in \overline{\mathbb{Q}}_{\ell}$ are algebraic over $\mathbb{Q}$, and the numbers $\ell, x_{1}, \ldots, x_{n}$ are multiplicatively independent. Then their $\ell$-adic logarithms $\log x_{1}, \ldots, \log x_{n}$ are algebraically independent over $\mathbb{Q}$.

Also, we shall use a similar particular case of the Archimedean Shanuel conjecture.

Conjecture 2. Let $x_{1}, \ldots, x_{n} \in \mathbb{C}$ be complex numbers algebraic over $\mathbb{Q}$ and multiplicatively independent. Then their logarithms $\ln x_{1}, \ldots, \ln x_{n} \in \mathbb{C}$ are algebraically independent over $\mathbb{Q}$.

Now we recall the definition of the regulator $\mathfrak{R}_{\ell}(K)$. As in [4], let $D(K)$ be a free

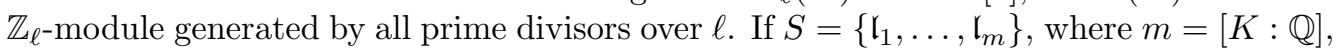

\footnotetext{
${ }^{1}$ The author is gratelul to Don Zagier who has pointed out this problem to the author.
} 
is the set of all such divisors, then any element of $D(K)$ may be represented uniquely in the form

$$
\sum_{i=1}^{m} a_{i} \mathfrak{l}_{i}, \quad a_{i} \in \mathbb{Z}_{\ell}
$$

If $u \in U_{S}(K)$, then the element $u$ gives rise to its principal divisor $(u)$ of the form (11). In this case all the corresponding coefficients $a_{i}$ are in $\mathbb{Z}$. This correspondence extends by continuity to a homomorphism div: $U_{S}(K)[\ell] \rightarrow D(K)$, where $[\ell]$ means pro- $\ell$ completion. As was proved in 44, Proposition 3.1], if the Leopoldt conjecture is valid for $K$ and $\ell$, then the homomorphism div induces an injection div: $U_{S, 1}(K) \hookrightarrow D(K)$.

One can define a standard scalar product on the $\mathbb{Z}_{\ell}$-module $D(K)$ :

$$
\langle,\rangle: D(K) \times D(K) \longrightarrow \mathbb{Z}_{\ell}, \quad\langle x, y\rangle=\sum_{i=1}^{m} a_{i} b_{i},
$$

where $x, y \in D(K), \quad x=\sum_{i=1}^{m} a_{i} \mathfrak{l}_{i}, \quad y=\sum_{i=1}^{m} b_{i} \mathfrak{l}_{i}, \quad a_{i}, b_{i} \in \mathbb{Z}_{\ell}$.

The regulator $\mathfrak{R}_{\ell}(K)$ is defined as the determinant of the matrix of the bilinear form

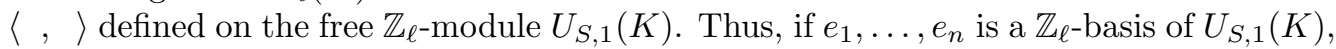
then

$$
\mathfrak{R}_{\ell}(K)=\operatorname{det}\left(\left\langle\operatorname{div}\left(e_{i}\right), \operatorname{div}\left(e_{j}\right)\right\rangle\right), \quad 1 \leq i, j \leq n .
$$

This definition was discussed in more detail in [4, §3].

So, we want to define some collection of $\mathbb{Z}_{\ell}$-bases in $U_{S, 1}(K)$ such that the passage from any basis of this collection to another basis can be done by using some transition matrix from $\operatorname{GL}(\mathrm{n}, \mathbb{Z})$. Then we would be able to define $\mathfrak{R}_{\ell}(K)$ by formula (2), taking for the basis $e_{1}, \ldots, e_{n}$ one of the bases of our collection.

First, we consider the case where $K$ is normal over $\mathbb{Q}$. In this case the field $K$ is either totally real or pure imaginary. We shall treat explicitly the latter case only, because the former can be treated in the same way. As in [4, §3], in our constructions we shall use an element $\alpha \in U_{S}(K)$ such that $(\alpha)=\mathfrak{l}_{1}^{\mathbf{h}}$, where $\mathfrak{l}_{1}$ is a prime divisor of $\ell$ in $K$, and $\mathbf{h}$ is the order of $\mathfrak{l}_{1}$ in the class group of $K$.

Obviously, the condition $(\alpha)=\mathfrak{l}_{1}^{\mathbf{h}}$ determines $\alpha$ up to multiplication by any element of the group $U(K)$. Thus, our first goal is to determine the element $\alpha$ canonically. For this, we shall use the homomorphism of the group $K^{\times}$into the logarithmic space $\mathbb{R}_{n}$ that was described in detail in [1, Chapter. 2]. Presently, we assume that $K$ is pure imaginary, that is, $m=[K: \mathbb{Q}]=2 n$. Let $\sigma_{1}, \bar{\sigma}_{1}, \sigma_{2}, \bar{\sigma}_{2}, \ldots, \sigma_{n}, \bar{\sigma}_{n}$ be all injections of $K$ into $\mathbb{C}$, where $\sigma_{i}, \bar{\sigma}_{i}$ are complex conjugate injections for $i=1,2, \ldots, n$. Then with any element $x \in K^{\times}$we can associate a vector $l(x)=\left(l_{1}(x), \ldots, l_{n}(x)\right)$, where $l_{i}(x)=\ln \left|\sigma_{i}(x)\right|^{2}=\ln \left(\sigma_{i}(x) \bar{\sigma}_{i}(x)\right)$. The map $l: K^{*} \rightarrow \mathbb{R}^{n}$ is a homomorphism, and ker $l \cap U(K)$ coincides with the group $\mu(K)$ of all roots of unity in $K$. The homomorphism $l$ maps the group of units $U(K)$ onto some lattice of rank $n-1$ contained in the subspace $V \subset \mathbb{R}^{n}$ determined by the condition

$$
V=\left\{x=\left(x_{1}, \ldots, x_{n}\right) \in \mathbb{R}^{n} \mid \sum_{i=1}^{n} x_{i}=0\right\} .
$$

Obviously, the vector $l(\alpha)$ does not belong to $V$, because $\sum_{i=1}^{n} l_{i}(\alpha)=\ln (N(\alpha))=\ln \left(\ell^{\mathbf{h}}\right)$. In the space $\mathbb{R}^{n}$ we have the standard Eucledian norm such that

$$
|x|=\sqrt{x_{1}^{2}+\cdots+x_{n}^{2}} \text { for } x=\left(x_{1}, \ldots, x_{n}\right) \in \mathbb{R}^{n} .
$$

Let $\tau$ be an automorphism of $K$. Then

$$
|l(x)|=|l(\tau(x))|
$$


for any $x \in K$. Indeed, if $\sigma_{i}$ and $\bar{\sigma}_{i}$ are complex conjugate, then so are $\sigma_{i} \tau$ and $\bar{\sigma}_{i} \tau$, that is, the vectors $x$ and $\tau(x)$ have the same coordinates up to permutation.

For a set $T \subset \mathbb{C}$, finite or infinite, we denote by $\ln T$ the set $\{\ln t \mid t \in T\}$. Respectively, for a field $k \subset \mathbb{C}$ by $k(\ln T)$ we denote the field generated over $k$ by the elements $\ln t$ for all $t \in T$.

Proposition 3. Assume that Conjecture 2 holds true. Let $K$ be a pure imaginary field normal over $\mathbb{Q}$ of degree $m=2 n$. Then the field $L:=\mathbb{Q}(\ln U(K))$ is of trancendence degree $r=n-1$ over $\mathbb{Q}$ and $M:=\mathbb{Q}\left(\ln U_{S}(K)\right)$ is of trancendence degree $r+m$ over $\mathbb{Q}$. Let $\alpha$ be as above, and let $\sigma_{1}, \ldots, \sigma_{m}$ be all automorphisms of $K$. Then the elements $\sigma_{1}(\alpha), \ldots, \sigma_{m}(\alpha)$ are algebraically independent over $L$.

Proof. Let $\varepsilon_{1}, \ldots, \varepsilon_{r}$ be a fundamental system of units in $U(K)$. Then by Conjecture 2 the elements $\ln \varepsilon_{1}, \ldots, \ln \varepsilon_{r}$ are algebraically independent over $\mathbb{Q}$, that is, $\operatorname{deg} \operatorname{tr}(L) \geq r$. Note that any unit $u \in U(K)$ can be represented (up to multiplication by some root of unity) in the form $u=\prod_{i=1}^{r} \varepsilon_{i}^{s_{i}}$ for some $s_{i} \in \mathbb{Z}$. Therefore, $\ln u=\sum_{i=1}^{r} s_{i} \ln \varepsilon_{i}$ and $L$ is a pure trancendental extension of $\mathbb{Q}$ generated by the elements $\ln \varepsilon_{1}, \ldots, \ln \varepsilon_{r}$. The elements $\varepsilon_{1}, \ldots, \varepsilon_{r}, \sigma_{1}(\alpha), \ldots, \sigma_{m}(\alpha)$ are multiplicatively independent and generate a subgroup of finite index in $U_{S}(K)$. Hence, their logarithms are algebraically independent and generate the field $M$ over $\mathbb{Q}$. This proves the proposition.

Theorem 4. Assume that Conjecture 2 holds true. Let $K$ be a normal extension of $\mathbb{Q}$, and suppose that the prime $\ell$ splits completely in $K$. Let $\mathfrak{l}_{1}$ be a fixed prime divisor of $\ell$ in $K$, and let $\alpha \in U_{S}(K)$ be an element such that $(\alpha)=\mathfrak{l}_{1}^{\mathbf{h}}$, where $\mathbf{h}$ is the order of $\mathfrak{l}_{1}$ in the class group. Put

$$
X(\alpha)=\left\{\alpha u \in K^{\times} \mid u \in U(K)\right\} .
$$

Then in the set $X(\alpha)$ there is a unique vector $\widetilde{\alpha}$ of minimal length. Let $\mathfrak{l}_{i}$ be another prime divisor of $\ell$ in $K$ and $\sigma_{i}$ an automorphism of $K$ such that $\sigma_{i}\left(\mathfrak{l}_{1}\right)=\mathfrak{l}_{i}$. Let $\widetilde{\alpha}_{i} \in K$ be an element constructed in the same manner as $\widetilde{\alpha}$ but relative to the prime divisor $\mathfrak{l}_{i}$. Then $\sigma_{i}(\widetilde{\alpha})=\widetilde{\alpha}_{i}$.

Proof. We have $|l(\alpha u)|=|l(\alpha)+l(u)| \geq|l(u)|-|l(\alpha)|$. Hence, $|l(\alpha u)|>|l(\alpha)|$ if $|l(u)|>$ $2|l(\alpha)|$. Therefore, it suffices to search the minimal value of $|l(\alpha u)|$ on some finite subset of $l(U(K))$, which proves the existence of this minimal value. Let $\left|l\left(\alpha u_{1}\right)\right|$ be this minimal value. We need to check that for any $u_{2} \in U(K)$ the condition $\left|l\left(\alpha u_{1}\right)\right|=\left|l\left(\alpha u_{2}\right)\right|$ implies $u_{1}=u_{2}$. Indeed, if $\left|l\left(\alpha u_{1}\right)\right|=\left|l\left(\alpha u_{2}\right)\right|$, then

$$
\begin{aligned}
\left|l\left(\alpha u_{1}\right)\right|^{2} & \left.=\sum_{i=1}^{n}\left(l_{i}\left(\alpha u_{1}\right)\right)^{2}=\sum_{i=1}^{n}\left(l_{i}(\alpha)+l_{i}\left(u_{1}\right)\right)\right)^{2} \\
& =\sum_{i=1}^{n}\left(l_{i}(\alpha)^{2}+2 l_{i}(\alpha) l_{i}\left(u_{1}\right)+l_{i}\left(u_{1}\right)^{2}\right) \\
& =\left|l\left(\alpha u_{2}\right)\right|^{2}=\sum_{i=1}^{n}\left(l_{i}(\alpha)^{2}+2 l_{i}(\alpha) l_{i}\left(u_{2}\right)+l_{i}\left(u_{2}\right)^{2}\right) .
\end{aligned}
$$

Thus, (5) yields the identity

$$
\sum_{i=1}^{n} 2\left(l_{i}\left(u_{1}\right)-l_{i}\left(u_{2}\right)\right) l_{i}(\alpha)+\sum_{i=1}^{n}\left(l_{i}\left(u_{1}^{2}\right)-l_{i}\left(u_{2}^{2}\right)\right)=0 .
$$

This gives an algebraic relation over $L$ among the $l_{i}(\alpha)$. On the other hand, we have $l_{i}(\alpha)=\ln \left(\sigma_{i}(\alpha) \bar{\sigma}_{i}(\alpha)\right)$, where $\sigma_{i}$ and $\bar{\sigma}_{i}$ are complex conjugate injections. So, the algebraic independence of the elements $\ln \sigma_{1}(\alpha), \ldots, \ln \sigma_{m}(\alpha)$ over $L$ stated in Proposition 3 
implies the algebraic independence over $L$ of the elements $l_{i}(\alpha), i=1, \ldots, n$. Thus, we obtain $l_{i}\left(u_{1}\right)=l_{i}\left(u_{2}\right)$ for $i=1,2, \ldots, n$, that is, $u_{1}$ and $u_{2}$ coincide up to multiplication by a root of unity. This proves the uniqueness of $\widetilde{\alpha}$.

Now suppose that, acting as above, we have constructed elements $\widetilde{\alpha}=\widetilde{\alpha}_{1}$ and $\widetilde{\alpha}_{i}$, where the former corresponds to the prime divisor $\mathfrak{l}_{1}$ and the latter to $\mathfrak{l}_{i}$. Suppose that the automorphism $\sigma_{i}$ sends $\mathfrak{l}_{1}$ to $\mathfrak{l}_{i}$. Then the element $\beta:=\sigma_{i}\left(\widetilde{\alpha}_{1}\right)$ belongs to $\widetilde{\alpha}_{i} U(K)$, and (4) shows that $|l(\beta)|$ is minimal on this set. Therefore, $\beta=\widetilde{\alpha}_{1}$ up to multiplication by a root of unity. This completes the proof of the theorem.

\section{§3. Definition And examples}

Let $K$ be normal over $\mathbb{Q}$. Suppose that $\ell$ splits completely in $K$, and that $\sigma_{1}, \ldots, \sigma_{m}$ are all injections of $K$ into $\mathbb{Q}_{\ell}$. Then any $x \in K^{\times}$gives rise to a vector $\sigma_{1}(x), \ldots, \sigma_{m}(x) \in$ $K \otimes_{\mathbb{Q}} \mathbb{Q}_{\ell} \cong \mathbb{Q}_{\ell}^{m}$ and, applying the $\ell$-adic logarithm map, we obtain a homomorphism

$$
\log _{\ell}: U_{S}(K)[\ell] \longrightarrow \mathbb{Q}_{\ell}^{m}, \quad \log _{\ell}(u)=\left(\log \sigma_{1}(u), \ldots, \log \sigma_{m}(u)\right)
$$

for any $u \in U_{S}(K)[\ell]$. This homomorphism was considered already in [4. As in that paper, we assume that the following conjecture holds true.

Conjecture 5. Let $\widetilde{\alpha}$ be the same as in $\S 2$. Then any $m-1$ among the elements

$$
\log _{\ell} \sigma_{1}(\widetilde{\alpha}), \ldots, \log _{\ell} \sigma_{m}(\widetilde{\alpha})
$$

are linear independent over $\mathbb{Q}_{\ell}$.

Note that $N_{K / \mathbb{Q}}(\widetilde{\alpha})=\ell^{\mathbf{h}}$, hence,

$$
0=\log _{\ell} N_{K / \mathbb{Q}}(\widetilde{\alpha})=\sum_{i=1}^{m} \log \sigma_{i}(\widetilde{\alpha}) .
$$

This conjecture (in a more general form) was formulated in [4] as Conjecture 3.1. In that paper (see [4, Proposition 3.4]), it was proved that this conjecture is a consequence of our Conjecture 1, which appeared in [4] as Conjecture 2.1.

As in [4, let $U_{S, 2}(K)$ be the subgroup of local universal norms in $U_{S}(K)[\ell]$. Then $U_{S, 1}(K) \subseteq U_{S, 2}(K)$ and, as it was proved in [4, (3.7)], the group $U_{S, 2}(K)$ consists of all $x \in U_{S}(K)[\ell]$ such that $\log _{\ell}(x)=0$. In [4, Proposition 3.5], it was shown that the group $U_{S, 1}(K)$ is of finite index in $U_{S, 2}(K)$ under the assumption that the Leopoldt conjecture and Conjecture 5 hold true. Thus, the last condition is satisfied if Conjecture 1 of the present paper is valid. In particular, using [4, Theorem 2.1], we see that $U_{S, 2}(K)$ is a free $\mathbb{Z}_{\ell}$-module of rank $r+1$, where $r=m / 2-1$ is the rank of the group of units of $K$.

Put

$$
\begin{aligned}
& U_{S, 1}^{0}(K)=\left\{x \in U_{S, 1}(K) \mid N_{K / \mathbb{Q}}(x)=1\right\}, \\
& U_{S, 2}^{0}(K)=\left\{x \in U_{S, 2}(K) \mid N_{K / \mathbb{Q}}(x)=1\right\} .
\end{aligned}
$$

Then, assuming Conjecture 1 to be true, we conclude that $U_{S, 1}^{0}(K)$ and $U_{S, 2}^{0}(K)$ are free

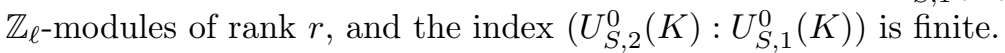

Now, let $\varepsilon_{1}, \ldots, \varepsilon_{r}$ be a fundamental system of units of $K$. Then, as in the proof of Proposition 3.5 in [4, we see that for any $i=1, \ldots, r$ there is a uniquely defined collection of coefficients $a_{i, j} \in \mathbb{Q}_{\ell}$ such that

$$
\sum_{j=1}^{m} a_{i, j} \log _{\ell} \sigma_{j}(\widetilde{\alpha})=\log _{\ell} \varepsilon_{i}, \quad \sum_{j=1}^{m} a_{i, j}=0,
$$

where $\widetilde{\alpha}$ is the canonical element constucted in Theorem 1 . 
Put

$$
\eta_{i}=\varepsilon_{i}^{-1} \prod_{j=1}^{m} \sigma_{j}(\widetilde{\alpha})^{a_{i, j}}
$$

Let $U_{S, 3}^{0}$ be a $\mathbb{Z}_{\ell}$-module generated by the elements $\eta_{1}, \ldots, \eta_{r}$. Then $\eta_{j}^{\ell^{d}} \in U_{S, 2}(K)$ if $d$ is so large that $\ell^{d} a_{i, j} \in \mathbb{Z}_{\ell}$ for all $i, j$. Therefore, the groups $U_{S, 3}^{0}(K)$ and $U_{S, 2}^{0}(K)$ are commensurable, that is, the index $\left(U_{S, 3}^{0}(K): U_{S, 2}^{0}(K)\right)$ is a well-defined integral power of $\ell$.

Note that in the group $U_{S, 3}^{0}(K)$ there is a special basis $\eta_{1}, \ldots, \eta_{r}$, which depends on the fundamental system of units $\varepsilon_{1}, \ldots, \varepsilon_{r}$. If one chooses another fundamental system of units $\varepsilon_{1}^{\prime}, \ldots, \varepsilon_{r}^{\prime}$ related to the system $\varepsilon_{1}, \ldots, \varepsilon_{r}$ via a transition matrix $C \in \operatorname{GL}(r, \mathbb{Z})$, and for the system $\varepsilon_{1}^{\prime}, \ldots, \varepsilon_{r}^{\prime}$ one constructs the elements $\eta_{1}^{\prime}, \ldots, \eta_{r}^{\prime}$ as above, then the elements $\eta_{1}^{\prime}, \ldots, \eta_{r}^{\prime}$ will be related to the elements $\eta_{1}, \ldots, \eta_{r}$ via the same transition matrix $C$. In particular, this means that the group $U_{S, 3}^{0}(K)$ is well defined, that is, it does not depend on the particular choice of the fundamental system of units. Now we put

$$
\mathfrak{R}_{\ell, 3}^{0}(K)=\mathfrak{R}_{\ell}^{0}\left(U_{S, 3}^{0}(K)\right)=\operatorname{det}\left(\left\langle\operatorname{div}\left(\eta_{i}\right), \operatorname{div}\left(\eta_{j}\right)\right\rangle\right), \quad 1 \leq i, j \leq r .
$$

So, after the passage from $\eta_{1}, \ldots, \eta_{r}$ to $\eta_{1}^{\prime}, \ldots, \eta_{r}^{\prime}$, the number $\mathfrak{R}_{\ell, 3}^{0}(K)$ is multiplied by $(\operatorname{det} C)^{2}=1$, that is, this number is well defined.

Now we put $U_{S, 3}(K)=U_{S, 3}^{0} \times \ell^{\mathbb{Z}_{\ell}}$. Thus, $U_{S, 3}(K)$ is a multiplicative free $\mathbb{Z}_{\ell \text {-module }}$ generated by the elements $\eta_{1}, \ldots, \eta_{r}, \ell$, and from (77) it follows that $\left\langle\operatorname{div}\left(\eta_{i}\right),(\ell)\right\rangle=0$ if $i=1, \ldots, r$. Taking the relation $\langle(\ell),(\ell)\rangle=[K: \mathbb{Q}]=m$ into account, finally we put

$$
\mathfrak{R}_{\ell, 3}(K)=\mathfrak{R}_{\ell, 3}^{0}(K) m, \quad \mathfrak{R}_{\ell}(K)=\mathfrak{R}_{\ell, 3}(K)\left(U_{S, 1}(K): U_{S, 3}(K)\right)^{2},
$$

where $\left(U_{S, 1}(K): U_{S, 3}(K)\right)$ is some integral power of $\ell$.

Now we assume that $k$ is an arbitrary algebraic number field, and that the prime $\ell$ splits completely in $k$. Let $K$ be the Galois closure of $k$ over $\mathbb{Q}$. Then all results obtained above are valid for the field $K$. In particular, the group $U_{S, 3}^{0}(K)$ is defined. We may assume that there is some fixed $\mathbb{Z}_{\ell}$-basis $\eta_{1}, \ldots, \eta_{r}$ of this group, which corresponds to the fundamental system of units $\varepsilon_{1}, \ldots, \varepsilon_{r}$ of $K$. Let $\epsilon_{1}, \ldots, \epsilon_{s}$ be a fundamental system of units of $k$. Then there is a representation

$$
\epsilon_{i}=\prod_{k=1}^{r} \varepsilon_{k}^{b_{i, k}}, \quad 1 \leq i \leq s, \quad b_{i, k} \in \mathbb{Z},
$$

whose coefficients $b_{i, k}$ are defined uniquely. We put $\xi_{i}=\prod_{k=1}^{r} \eta_{k}^{b_{i, k}}, i=1, \ldots, s$. Then from (8) and (11) it follows that $\xi_{i}=\epsilon_{i}^{-1} A_{i}$ for $i=1, \ldots, s$, where

$$
A_{i}=\prod_{k=1}^{r}\left(\prod_{j=1}^{m} \sigma_{j}(\widetilde{\alpha})^{a_{k, j}}\right)^{b_{i, k}} .
$$

Let $H$ be the subgroup corresponding to $k$ in the Galois group $G=G(K / \mathbb{Q})$. Then the element $\log _{\ell} A_{i}=-\log _{\ell} \epsilon_{i}$ is fixed under the action of $H$. The element $A_{i}$ can be written uniquely in the form $A_{i}=\prod_{j=1}^{m} \sigma_{j}(\widetilde{\alpha})^{c_{i, j}}$ with $c_{i, j} \in \mathbb{Q}_{\ell}$, and (7) implies that $\sum_{j=1}^{m} c_{i, j}=0$ for $i=1, \ldots, s$. Thus, the divisor $\mathfrak{A}_{i}=\operatorname{div}\left(\xi_{i}\right)=\operatorname{div}\left(A_{i}\right)$ is fixed under the action of $H$. Therefore, there is $d \in \mathbb{Z}$ such that (in additive notation) $\ell^{d} \mathfrak{A}_{i} \in D(k)$. Put $t=|H|=[K: k]$. Denoting the pairing $\langle$,$\rangle in the fields K$ and $k$ by $\langle,\rangle_{K}$ and $\langle,\rangle_{k}$, respectively, we have $\langle x, y\rangle_{k}=t\langle x, y\rangle_{K}$ for any $x, y \in D(k)$. 
We define $U_{S, 3}^{0}(k)$ to be the $\mathbb{Z}_{\ell}$-module generated by the elements $\xi_{i}, i=1, \ldots, s$, and put

$$
\mathfrak{R}_{\ell, 3}^{0}(k)=\operatorname{det}\left(\left\langle\operatorname{div}\left(\xi_{i}\right) \operatorname{div}\left(\xi_{j}\right)\right\rangle_{K}\right)=t^{-1} \operatorname{det}\left(\left\langle\operatorname{div}\left(\xi_{i}\right) \operatorname{div}\left(\xi_{j}\right)\right\rangle_{k}\right), \quad 1 \leq i, j \leq s .
$$

Then we put $U_{S, 3}(k)=U_{S, 3}^{0}(k) \times \ell^{\mathbb{Z}_{\ell}}$ and $\mathfrak{R}_{\ell, 3}(k)=\mathfrak{R}_{\ell, 3}^{0}(k)[k: \mathbb{Q}]$.

Finally, as in (10), we put

$$
\mathfrak{R}_{\ell}(k)=\mathfrak{R}_{\ell, 3}(k)\left(U_{S, 1}(k): U_{S, 3}(k)\right)^{2} .
$$

Note that in the case of an Abelian field $k$ we can avoid using Conjecture 1. Indeed, in this case the Leopoldt conjecture is proved, as well as Conjecture 5 (see [4, Proposition 3.3]).

Concerning Conjecture 2, the author has found the only nontrivial case where the assertion of Theorem 1 can be verified without using this conjecture.

Theorem 6. Let $K$ be a real quadratic field and $\ell$ a prime number splitting in $K$, $(\ell)=\mathfrak{l}_{1} \mathfrak{l}_{2}$. Let $\alpha \in U_{S}(K)$ be an element such that $(\alpha)=\mathfrak{l}_{1}^{\mathbf{h}}$, where $\mathbf{h}$ is the order of $\mathfrak{l}_{1}$ in the class group of $K$. Then the condition $\left|l\left(\alpha u_{1}\right)\right|=\left|l\left(\alpha u_{2}\right)\right|$ for some units $u_{1}, u_{2} \in U(K)$ implies $l\left(u_{1}\right)=l\left(u_{2}\right)$, that is, $u_{1}= \pm u_{2}$.

Proof. For any $x \in K^{\times}$the point $l(x)$ belongs to $\mathbb{R}_{2}$ and has coordinates $x_{1}=\ln \left|\sigma_{1}(x)\right|$ and $x_{2}=\ln \left|\sigma_{2}(x)\right|$, where $\sigma_{1}, \sigma_{2}$ are two different injections of $K$ into $\mathbb{R}$. In particular, $l(U(K))$ is a one-dimensional lattice on the line $L$ given by the equation $x_{1}+x_{2}=0$. If $\left|l\left(\alpha u_{1}\right)\right|=\left|l\left(\alpha u_{2}\right)\right|$, then, multiplying by $l\left(u_{1}^{-1} u_{2}^{-1}\right)$, we obtain $\left|l\left(\alpha u_{1}^{-1}\right)\right|=\left|l\left(\alpha u_{2}^{-1}\right)\right|$, i.e., $\left|l(\alpha)-l\left(u_{1}\right)\right|=\left|l(\alpha)-l\left(u_{2}\right)\right|$. This means that the triangle $\Delta$ with vertices $l(\alpha), l\left(u_{1}\right)$, and $l\left(u_{2}\right)$ is isosceles. Let $O=\left(l\left(u_{1}\right)+l\left(u_{2}\right)\right) / 2$ be the midpoint of the base $\left[l\left(u_{1}\right), l\left(u_{2}\right)\right]$. Then the segment $[l(\alpha), O]$ is a height of the triangle $\Delta$, that is, the vector $\overrightarrow{O l(\alpha)}$ is orthogonal to the vector $(+1,-1)$, which is a directing vector of the line $L$. In its turn, this means that the coordinates of the vector $\overrightarrow{O l(\alpha)}$ are equal, i.e.,

$$
\ln \left|\sigma_{1}(\alpha)\right|-\frac{1}{2} \ln \left|\sigma_{1}\left(u_{1}\right)\right|-\frac{1}{2} \ln \left|\sigma_{1}\left(u_{2}\right)\right|=\ln \left|\sigma_{2}(\alpha)\right|-\frac{1}{2} \ln \left|\sigma_{2}\left(u_{1}\right)\right|-\frac{1}{2} \ln \left|\sigma_{2}\left(u_{2}\right)\right| .
$$

Since $\ln \left|\sigma_{2}\left(u_{1}\right)\right|=-\ln \left|\sigma_{1}\left(u_{1}\right)\right|$ and $\ln \left|\sigma_{2}\left(u_{2}\right)\right|=-\ln \left|\sigma_{1}\left(u_{2}\right)\right|$, from the preceding we obtain the equation

$$
\ln \left|\sigma_{1}(\alpha)\right|-\ln \left|\sigma_{2}(\alpha)\right|-\ln \left|\sigma_{1}\left(u_{1}\right)\right|-\ln \left|\sigma_{1}\left(u_{2}\right)\right|=0 .
$$

After exponentiation we get

$$
\beta:=\sigma_{1}(\alpha) \sigma_{2}(\alpha)^{-1} \sigma_{1}\left(u_{1}\right)^{-1} \sigma_{1}\left(u_{2}\right)^{-1}= \pm 1,
$$

but this is impossible because $\beta$ is an algebraic number with a divisor $\mathfrak{l}_{1}^{\mathbf{h}} \mathfrak{l}_{2}^{-\mathbf{h}}$. This proves the theorem.

\section{RefEREnCES}

[1] Z. I. Borevič and I. R. Šafarevič, The theory of numbers, Nauka, Moscow, 1964; English transl., Newcomb Greenleaf Pure Appl. Math., vol. 20, Acad. Press, New York, 1966. MR0195803

[2] S. Lang, Cyclotomic fields. I, II, Comb. 2nd ed., Grad. Texts in Math., vol. 121, Springer-Verlag, New York, 1990. MR1029028

[3] L. V. Kuz'min, Some remarks on an $\ell$-adic Dirichlet theorem and $\ell$-adic regulator, Izv. Akad. Nauk SSSR Ser. Mat. 45 (1981), no. 6, 1203-1240; English transl., Math. USSR-Izv. 19 (1982), no. 3, 445-478. MR641800

[4] - On a new type of $\ell$-adic regulator for algebraic number fields (the $\ell$-adic regulator without logarithms), Izv. Ross. Akad. Nauk Ser. Mat. 79 (2015), no. 1, 115-152; English transl., Izv. Math. 79 (2015), no. 1, 109-144. MR 3352584 
[5] - Some remarks on the $\ell$-adic regulator. V. The growth on the $\ell$-adic regulator in the cyclotomic $\mathbb{Z}_{\ell}$-extension of an algebraic number field, Izv. Ross. Akad. Nauk Ser. Mat. 73 (2009), no. 5, 105-170; English transl., Izv. Math. 73 (2009), no. 5, 959-1021. MR2584230

National Research Center "Kurchatovskit Institute", Akad. Kurchatov Sq. 1, 123182 MosCOW, Russia

E-mail address: 1vkuzmin@mail.ru

Received 25/JUN/2015

Translated by THE AUTHOR 